\title{
12 THESEN
}

\section{Zusammenspiel von Führung und Aufsicht}

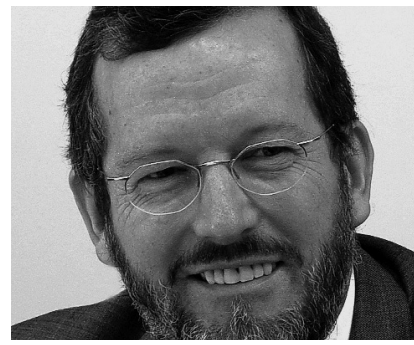

VON MARTIN BECK

Prof. Martin Beck, Großhandelskaufmann und Diplombetriebswirt (FH), war Geschäftsführer im Diakonischen Werk Württemberg, Gründer und Geschäftsführer einer Beratungsgesellschaft und Sanierer in der Industrie. Er ist jetzt tätig als selbstständiger Unternehmensberater, Autor, Aufsichtsrat und Hochschullehrer.

Internet http://www.prof-beck.net

\author{
Die Trennung von Leitung und Aufsicht ist eine der \\ wichtigsten Forderung für eine gute Unternehmens- \\ führung. Das Zusammenspiel von Führung und Aufsicht \\ muss immer wieder neu bedacht werden - für die So- \\ zialwirtschaft im Allgemeinen und für die je besondere \\ Organisation, um die es geht.
}

Die Themen Aufsicht und Führung sind in Bewegung geraten. Der Gesetzgeber setzt neue Schwerpunkte, die Diskussion um Corporate Governance bringt viele Gremien zum Nachdenken, und die Frage der Haftung bewegt manche Gremienmitglieder. Das ist gut so, denn die Sozialwirtschaft litt lange an einer schleichenden Entwertung der Aufsichtsgremien und einer latenten Alleinherrschaft der Führung.

\section{These: Alleinherrschaft ist nicht wünschenswert!}

Das Leninsche Einzelleitungsprinzip, nach dem machtvolle Einzelfiguren installiert werden und Kontrolle von oben nach unten stattfinden sollte, hat sich nicht überall bewährt. Macht verändert den Menschen, und zwar ausnahmslos jeden. Die Ausübung von Macht durch wenig kontrollierte und kontrollierbare Einzelpersonen kann für diese lustbringend sein, aber sie birgt die permanente Versuchung, die mit der Beherrschung von Menschen verbunden ist. Macht verdirbt den Charakter mehr als Geld. Alleinherrscher verlieren leicht den Kontakt zum wirklichen Leben. Deshalb spricht Vieles (wenngleich nicht alles) für die Installierung von Leitungsorganen aus zwei oder mehr Personen.

\section{These: Auch der Klügste braucht Korrektur!}

Allein jagt sich's zwar am besten, aber vier Augen sehen mehr als zwei. Und weil jedes Problem mehr Aspekte hat, als man auf den ersten Blick erkennt, spricht vieles für eine Führung aus mehr als einer Person. Korrektur ist sozusagen institutionell gesichert. Das ist sehr beruhigend. Eine Zweier- oder Dreierführung löst dynamische Prozesse aus, die dem Geschäft durchaus zugutekommen können. Man passt aufeinander auf, man kann sich gegenseitig helfen, man kann Geschlossenheit demonstrieren und man kann sich auf gleichem Level vertreten.

\section{These: Wer berichten muss, muss denken!}

Manchmal ist es richtig lästig, regelmäßig in mündlicher oder schriftlicher Form an Außenstehende berichten zu müssen, was sich ereignet hat, was sich aktuell bewegt - oder nicht und warum nicht -, und welche Vorhaben gelungen oder misslungen sind. Rechenschaftspflichten sind aber heilsam. Wer seine Arbeit regelmäßig rechtfertigen muss, der muss sie regelmäßig überdenken. Das hilft, nicht nur denjenigen, denen Bericht erstattet wird, sondern auch dem Berichterstatter selbst. Denken ist immer begrüßenswert, auch wenn es nicht ganz freiwillig geschieht.

\section{These: Mit anderer Leute Geld muss man transparent arbeiten!}

Die meisten Führungskräfte arbeiten mit anvertrautem Gut. Es ist nicht das 
eigene Geld, sondern das Geld von bekannten oder anonymen Investoren, Aktionären, Mitgliedern, Spendern oder Gesellschaftern. Sie alle haben ein Recht auf Transparenz. Wird diese Transparenz freiwillig und ohne Aufforderung hergestellt, dann entsteht Vertrauen. Muss diese Transparenz erst erzwungen werden, dann entsteht Misstrauen. Eigentlich erstaunlich, dass dieses Thema überhaupt diskutiert werden muss. Die Finger, mit denen es in der Sozialwirtschaft angefasst wird, sind manchmal sehr spitz. Wir haben doch nichts zu verbergen, oder?

\section{These: Unabhängiger Rat ist am Wertvollsten!}

Es ist schön, guten Rat von Freunden, von Kollegen, von Brüdern und Schwestern zu bekommen. Es ist besser, guten Rat auch von Leuten zu holen, die weder durch geschäftliche Abhängigkeiten noch durch freundschaftliche Bande daran gehindert sind, die Wahrheit in aller Klarheit auszusprechen. Ein solcher unabhängiger Rat kann schmerzhaft sein. Aber er kann Sie oder Ihr Werk retten. Solch einen Rat kann man sich auch kaufen - aber nur von Leuten, die von diesem Auftrag nicht abhängig sind. Da lohnt sich die offene Nachfrage bei Ihren Beratern.

\section{These: Guter Rat ist (nicht) teuer!}

Sozialwirtschaftliche Führungskräfte verhalten sich zum Thema Beratung genau so widersprüchlich, wie ihre Kollegen vom Profitlager. Die meisten sind eher beraterkritisch eingestellt, obwohl sie nie mit einem guten Berater gearbeitet haben. Manche sind beraterhörig, obwohl sie damit ihre eigene Position infrage stellen. Dabei liegt die Wahrheit hier, wie häufiger im Leben, eher in einer maßvollen Mittelposition. Dauerberatung: nein! Berater als Sündenbock: wenn's hilft, ja! Berater als Anreger, Kritiker, Korrektor und Motivator: gerne! Der teuerste Rat ist ja immer der, auf den ich nicht gehört habe.

\section{These: Aufsicht heißt auch Beratung und Fürsorge!}

Aufsicht klingt nach Distanz und Dis$\operatorname{tanz}$ ist eine gewichtige Voraussetzung für die erfolgreiche Tätigkeit in einem
Aufsichtsgremium. Aber Distanz ist nicht Feindschaft, sondern bewusstes Erkennen, Akzeptieren und Leben der satzungsmäßig vorgegebenen Rollen. Wer Aufsicht führt, ist nicht die Gremienpolizei. Beratung und Kontrolle markieren traditionell die beiden Extrempositionen, zwischen denen sich Aufsichtsgremien bewegen. Sie sind, wenn es gutgeht, zugleich Berater und Korrektiv. Aufsichtsgremien tragen auch die Verantwortung dafür, dass es den Führungskräften gutgeht. Nicht nur geschäftlich, sondern umfassend. Denn nur Führungskräfte, die sich gut aufgenommen, gut aufgehoben und vertrauensvoll begleitet erleben, sind nachhaltig und mit langem Atem erfolgreich.

\section{These: Auch Aufsicht ist nicht unfehlbar!}

Wer das letzte Wort hat, ist nicht hinterfragbar und damit quasi unfehlbar. Das ist für die Betroffenen angenehm, entspricht aber nicht der menschlichen Erfahrung. Auch Aufsichtsgremien können sich irren. Es ist keine Schande, einen falschen Kurs zu korrigieren. Im Gegenteil: Wer einen Irrtum offen zugibt und seinen Kurs tatkräftig korrigiert, der zeigt seine Lernbereitschaft und seine Beweglichkeit. Sturköpfe und Rechthaber sind auf Dauer mit Vorsicht zu genießen. Deshalb, liebe Aufsichtsratskollegen: Ruhig auch mal einen kritischen Blick auf die eigene Entscheidungskarriere werfen!

\section{These: Aufsicht braucht Distanz und Nähe!}

Es ist doch so schön, wenn sich alle mögen! Die Vorstände und die Aufsichtsräte und die Vereinsmitglieder und die Mitarbeiter - und die Gatten treffen sich auch noch im Kindergarten oder im Fitnessstudio. Das macht doch vieles einfacher - denkt man. Ohne Nähe kein Vertrauen, ohne Vertrauen keine konfliktfreie Zusammenarbeit. So weit so gut. Die Wahrheit ist: Wo keine Distanz da ist, da ist auch keine wirkliche Aufsicht möglich! Führung und Aufsicht haben mit Macht zu tun. Macht muss in einem demokratischen Gemeinwesen transparent, befristet und begrenzt sein. Machtbalance braucht Rollensicherheit. Nur mit klaren Rollen lassen sich Führung und Aufsicht wirksam ausüben.

\section{These: Der Wegweiser bleibt an der Kreuzung stehen!}

Gute Ratgeber gehen ein Stück weit den Weg ihrer Kunden mit, aber sie bleiben irgendwann zurück. Sonst würden sie ja die Seiten und die Rollen wechseln. Dann kann es sein, dass die Führungskraft den Weg alleine, vielleicht sogar mutterseelenallein, weiter gehen muss. Der Weg nach vorne ist oft einsam, weil die Meute erst überzeugt werden muss, dass Bewegung die Grundlage der Existenzsicherung ist. Gute Ratgeber bleiben zurück, beobachten aufmerksam, stehen bereit aber sie mischen sich nicht aktiv ein. Ratgeber, die immer mitgehen wollen, haben ihre Rolle nicht verstanden.

\section{These: Transparenz schafft Vertrauen!}

Nur harmlose Menschen glauben, dass immer alles öffentlich verhandelt werden müsse. Öffentlichkeit kann in frühen oder in kritischen Phasen eines Prozesses tödlich sein. Zu früh geplappert, zu früh gefragt, zu früh einbezogen - das heißt häufig: totgeredet. Aber trotzdem ist Transparenz ein wichtiges Vertrauensgut im Unternehmen. Nur was offen gelegt wird, kann auch nachvollzogen werden. Nur was ich verstehe, kann ich vertreten. Transparenz bedeutet »Durchblick «. Wer diese Transparenz fürchtet oder sie gar verweigert, der macht sich angreifbar und bringt sich in missverständliche Situationen.

\section{These: Nase rein und Finger raus!}

Das komplizierte und komplexe Verhältnis von Führung und Aufsicht lässt sich auf diese simple Formel reduzieren. Nase rein heißt: Aufsichtsgremien müssen fragen, fragen, fragen. Und Finger raus heißt: Die Rollen müssen klar bleiben. Aufsicht hat im operativen Geschäft nichts verloren! Das Wichtigste ist, dass jeder seine Rolle kennt. Laienspieler sind nicht rollensicher und verirren sich öfters. Profis kennen ihre Rolle und sind für diskrete Korrekturen dankbar. 